Kindes ist (beispielsweise der Stiefvater), nicht Beschuldigter ist oder wenn es sich bei dem Beschuldigten um einen nahen Verwandten handelt. Mit Rie $\beta^{39}$ ist hier eine Analogie zu $₫ 5_{2}$ Abs. 2 StPO anzunehmen, da der Gesetzgeber mit der Norm die Fälle regeln wollte, in denen der nicht beschuldigte Partner in der Ausübung des Zeugnisverweigerungsrechts in einen Interessenkonflikt gerä $\mathrm{t}^{4 \circ}$ und ein solcher auch bei diesen Konstellationen anzunehmen ist.

\title{
IV. Resümee
}

Historisch wurden Verletzteninteressen aus dem Strafprozeß verdrängt. Die Reformen der letzten Jahre, das Opferschutzgesetz, das Gesetz für die Entschädigung von Opfern von Gewalttaten und der Täter-Opfer-Ausgleich, haben bereits eine legislative Grundlage geschaffen, damit die Opferinteressen vermehrt im Prozeß berücksichtigt werden können. Dem ZSchG liegt der Gedanke zugrunde, dem Anspruch des Verletzten auf ein faires Verfahren und Wahrung seiner berechtigten Interessen Rechnung zu tragen. ${ }^{{ }^{\mathrm{I}}}$ Für über sechzehnjährige Zeugen ist der Gesetzesentwurf der Bundesregierung sicherlich ein weiterer Schritt in Richtung Opferschutz, der speziellen Situation kindlicher und jugendlicher Zeugen wird er jedoch nicht gerecht.

\section{Michael Maier-Borst Folter - Außenpolitische Ächtung versus innenpolitische
"Zurückhaltung « in der Bundesrepublik}

\section{Einleitung}

Ein Spannungsverhältnis zwischen Innen- und Außenpolitik festzustellen oder Inkonsistenzen in einem der beiden Felder zu markieren, gehört zum alltäglichen Geschäft der Opposition, der Nichtregierungsorganisationen und der Sozialwissenschaften. Der Aufsatz versucht, Probleme der wirksamen Bekämpfung bzw. Ächtung von Folter durch Politik und Recht in der Bundesrepublik Deutschland nachzuzeichnen. Anfangs sollen kurz die Ansatzpunkte im internationalen Recht für die Arbeit von amnesty international (ai) gegen Folter sowie insbesondere das Engagement der Organisation für den Schutz von politischen Flüchtlingen erläutert werden. Danach wird einerseits der vermeintliche gesellschaftliche Konsens in der Bundesrepublik hinterfragt, der vorgibt, Folter sei umfassend geächtet, andererseits sollen abschließend mit Blick auf die asylrechtliche Seite von Folter einige Probleme in der Betreuungsarbeit von gefolterten Flüchtlingen aufgezeigt werden.

39 Ohne wetcere Begrundung in NJW 1975, S. 83.

40 BT-Drs. $7 / 551$, S. $59 \mathrm{f}$.

4 I BT-Drs. $13 / 7165$, S. 4 
Folter gehört - wie die Todesstrafe - zu denjenigen Menschenrechtsverletzungen, gegen die sich ai in jedem Fall wendet. ai sieht Folter als eine Form von grausamer, unmenschlicher und erniedrigender Behandlung an und bezieht sich dabei u.a. auf das Folterverbot des Artikel s der Allgemeinen Erklärung der Menschenrechte vom 10. I 2. 1948, auf Art. 7 des Internationalen Paktes über bürgerliche und politische Rechte vom 19. 12. I 966 und auf die Antifolterkonvention der Vereinten Nationen aus dem Jahre i975 (UN-Konvention gegen Folter und andere unmenschliche, grausame oder erniedrigende Behandlung oder Strafe vom 9. 12. 1975). ${ }^{1}$ "Folter " wird in der UN-Antifolterkonvention von 1975 in Art. I Absatz I wie folgt definiert:

"Unter Folter im Sinne dieser Erklärung st jede Handlung zu verstehen, durch die einer Person durch einen Träger staatlicher Gewalt oder auf dessen Veranlassung bin vorsätzlich starke körperliche und getstig-seelische Schmerzen oder Leiden zugefügt werden, um von thr oder einem Dritten eine Aussage oder ein Geständnis zu erzwingen, ste fü eine tatsächliche oder mutmaßliche von ihr begangene Tat zu bestrafen oder sie oder andere Personen einzuschüchtern."

In Satz 2 dieses Artikels wird die Definition jedoch empfindlich eingeschränkt. Dort heißt es:

"Nicht darunter (unter den Folterbegriff, M.M.-B.) fallen Schmerzen und Leiden, die sich lediglich in einem mut den Mindestbedingungen über die Behandlungen von Strafgefangenen zu vereinbarenden Maß aus gesetzluch zulässigen Zwangsmaßnabmen ergeben, diesen anhaften oder als deren Nebenwirkungen auftreten."

Ein Folterverbot mit individueller Beschwerdemöglichkeit war für die Staaten des Europarates zum Zeitpunkt der Verabschiedung der zitierten Formulierungen schon Realität: Art. 3 in Verbindung mit Art. 13 der Europäischen Konvention zum Schutze der Menschenrechte und Grundfreiheiten vom 4. I1. 1950 (EMRK) verbieten die Anwendung von Folter in den Unterzeichnerstaaten. Darüber hinaus führte das Europäische Übereinkommen zur Verhütung der Folter und unmenschlicher oder erniedrigender Behandlung oder Strafe vom 26. 1 I. I987 einen vorbeugenden Kontrollmechanismus ein, der die Anwendung von Folter offenlegen, erschweren und damit schließlich verhindern soll.

ai hat sich u. a. das Ziel gesetzt, die Abschiebung von Menschen in Länder zu verhindern, in denen ihnen Inhaftierung als gewaltlose politische Gefangene, ${ }^{2}$ Folter, Todesstrafe oder sogenanntes "Verschwindenlassen « drohen. Die beiden zu klärenden Fragen lauten: In welchem politischen und gesellschaftlichen Umfeld versucht ai, die Abschiebung in einen folternden Staat zu verhindern? Und daran anschließend: Erhalten Flüchtlinge, die Folter erlitten oder zu gewärtigen haben, in bundesdeutschen Asylverfahren den angemessenen dauerhaften Schutz vor Abschiebung?

\footnotetext{
I Die Antifolterkonvention wurde am 10. 12. 1984 von der Generalversammlung der Vereinten Nationen durch die Resolution 39/46 angenommen, sie isc seit dem 26.6.1987 volkerrechtlich in Kraft; fur die Bundesrepublik seit dem 31. 10.1990 (BGBl. 1990 II, S. 247 ff.).

2 Internationale Satzung von as Art. I : "ai wendet sich gegen schwerwegende Verletzungen des Rechtes eınes jeden Menschen auf Meinungsfreshett und freie Meınungsaußerung, auf Schutz vor Diskriminierung aufgrund semer ethnıschen Abstammung, seines Geschlechtes, seiner Hautfarbe oder seiner Sprache, se1ner nationalen oder sozialen Herkunft, seines okonomischen Status, seiner Geburt oder eines anderen Status, sowie auf korperlıche und geistıge Unversehrtheit. « Haben Personen aufgrund ihrer religiosen, politischen oder anderer gesstiger Uberzeugungen Inhaftierung zu erdulden, fordert die Organisation in den Fallen die sofortige Freilassung aus der Haft, in denen diese polıtischen Gefangenen weder Gewalt angewendet noch zu deren Anwendungen aufgerufen haben. Solche Gefangenen werden als "gewaltlose politische Gefangene bezeichnet.
} 
Die von der Bundesrepublik unterschriebenen internationalen Konventionen gegen Folter deuten auf einen breiten gesellschaftlichen Konsens gegen Folter hin. Und auch nach Durchsicht der Äußerungen einiger Politiker anläßlich des Inkrafttretens der Antifolterkonvention der Vereinten Nationen in der Bundesrepublik lassen sich deutliche Mißtöne kaum finden. ${ }^{3}$ Stellvertretend für viele sei hier aus der damaligen Bundestagsdebatte der CDU-Abgeordnete Seesing zitiert:

„Man muß sich fragen, ob das nicht Dinge sein müßten, von denen man gar nicht mehr redet, denn wer von uns will eigentlich Folter? (...) Die menschliche Gesellschaft ist eine Gemeinschaft von Personen, von Wesen also, die in ihrer Art einmalig, unverwechselbar, frei und nach meiner religiosen Auffassung unsterblich sind. Wegen dieser Eigenschaften hat ein Mensch das Recht auf die Unverletzlichkeit seiner Person. Es dient seiner Freiheit. Es dient der Sicherung seiner Eigenverantwortung. Es gibt nur ganz wenige Gründe, auf Grund eines Gesetzes in die Freiheitsrechte eines Menschen einzugreifen. Aber diese Eingriffe dürfen niemals die körperliche Unversehrtheit in Frage stellen." (Stenographische Berichte des Deutschen Bundestages, I I. Wahlperiode, 194. Sitzung vom 8. 2. 1990, S. 14902).

Solche klaren Worte hört man auch in der Bundesrepublik selten, wenn es um Menschenrechte geht. Schon bei dem Thema Todesstrafe kann man - inner- wie außerhalb des Parlaments - kaum einen ähnlichen gesellschaftlichen Konsens behaupten. Es ist bemerkenswert, daß Folter, zumindest vordergründig, erfolgreich geächtet werden konnte, ${ }^{4}$ Todesstrafe jedoch von vielen Menschen unter gewissen Umständen als »legitim« angesehen wird. Meine Vermutungen gehen dahin, daß zum einen die notwendige richterliche Anordnung der Todesstrafe, zum anderen die zwar falsche, aber offensichtlich wirksame Behauptung, es existierten humane oder weniger humane Arten der Todesstrafe, eine große Rolle für ihre gesellschaftliche Billigung spielen. Trotzdem erweist sich auch der Konsens über die grundsätzliche Ablehnung von Folter in Drucksituationen als brüchig. Oft werden in der Bundesrepublik ohne Not Chancen ausgelassen, dem absoluten Folterverbot des Völkerrechts Nachdruck zu verleihen. Hierzu sollen im folgenden Beispiele aus dem innenpolitischen Bereich gegeben werden.

3 Ganz ohne Auseinandersetzungen lief die Diskussion naturlich nicht ab. Neben der langen Zeitspanne zwischen Unterschrift und Inkrafttreten der Antifolterkonvention der Vereinten Nationen - die Bundesrepublik setzte die Konvention als einer der letzten westeuropaischen Staaten in Kraft - wurden dabeı die Isolationshaft und die sog. "Interpretationserklärung" der Bundesregierung zu Art. 3 thematisiert. Letztere sollte auf Druck der Bundeslander Bayern und Baden-Wurttemberg vor dem Hintergrund der damalıgen hitzigen Asyldiskussion unterstreichen, daß sich aus erlittener Folter nicht direkt ein Rechtsanspruch auf eine Eınresse- oder Aufenthaltserlaubnis ergibt. Der bayerische Innenmınister, Stoiber, befurchtete einen »neuen Asyltatbestand “ (vgl. Suddeutsche Zeitung 13.7. 1989 und Stenographische Berichte des Deutschen Bundestages, I 1. Wahlperiode, 194. Sitzung vom 8. 2. 1990 , S. $14902 \mathrm{ff}$.).

4 Winfried Brugger, der die Frage ,Darf der Staat ausnahmsweise foltern? $\times$ in der Zeitschrift Der Staat ( $1 / 96$, S. 67-97) mit einer Relativierung des hier unterstellten absoluten Folterverbots beantwortet, ist derzeit, soweit ich sehe, eine Ausnahme in der Zunft der Rechtswissenschaftler. Auch der Duktus seiner Überlegungen, der irgendwo zwischen den unsaglichen Zumutungen der Frage-Antwort-Spiele vergangener Jahre in bundesdeutschen Kreiswehrersatzamtern und akademischen Bemuhungen liegen mag, steht einzig da. Das Thema und seıne entsprechende Antwort einem Jubilar darzureichen, zeigt hoffentlich nur, daß man sich Gratulanten und deren Darreichungen nicht aussuchen kann. 
Aussagen, die in Strafprozessen als Beweis herangezogen werden sollen, dürfen nicht unter Folter herbeigeführt worden sein. Dies ist in der Literatur und in der Rechtspraxis der Bundesrepublik unbestritten (vgl. Art. is UN-Antifolterkonvention; $\$$ I $_{3}$ 6a Absatz 3 Satz 2 StPO).s Das Verwertungsverbot für durch Folter herbeigeführte Aussagen entfaltet neben dem schlichten Folterverbot zusätzlich eine präventive Wirkung: Folter soll sich niemals politisch oder strafrechtlich »lohnen «, da Aussagen, die unter Folter herbeigeführt wurden, grundsätzlich in keinem Verfahren verwertet werden dürfen.

Die 3. Kammer des 2. Senates des BVerfG hat hierzu 1996 in einer einstimmigen Entscheidung über die Nichtannahme einer Verfassungsbeschwerde, die ein Auslieferungsersuchen Spaniens gegenüber der Bundesrepublik betraf, schwer nachvollzichbare Aussagen gemacht. Thnen fehlt es an der gebotenen Deutlichkeit, wenn es um den präventiven Charakter der Antifolterkonvention, insbesondere ihres Art. is geht. Der Beschwerdeführer, ein mutmaßlicher ETA-Unterstützer, wandte sich u.a. gegen die Auslieferung nach Spanien, da er vor allem durch die Aussage eines ehemaligen Komplizen belastet werde, der die Anschrift einer Unterstützerwohnung preisgegeben habe, die der Beschuldigte angemietet hatte. Die Aussage des Komplizen sei unter Folter herbeigeführt worden. Die 3. Kammer bemerkt hierzu,

»daß mit der Auslieferung des Beschwerdeführers elementare Anforderungen des Rechtsstaates oder gar der völkerrechtliche Mindeststandard auch dann nicht verletzt würden, wenn die Anschrift der Wohnung (...) den spanischen Behorden (...) nur aufgrund einer Aussage bekannt wurde, die durch Folter eines anderen Verdächtigten herbeigefuhrt worden war. “ ${ }^{6}$

In dem Teil der Begründung, die sich mit durch Folter herbeigeführten Aussagen auseinandersetzte, wird daran anknüpfend zuerst eine generelle "Fernwirkung « auf Verfahren im Ausland verneint. Darüber hinaus sei eine Fernwirkung und damit der Art. is der Antifolterkonvention auf Verfahren in anderen Staaten dann eingeschränkt zu interpretieren, wenn sie auf Aussagen bezogen werden solle, die nicht der Beschuldigte selbst, sondern ein Dritter unter Folter gemacht habe. Eine inhaltliche Auseinandersetzung mit dem vorgebrachten Problem von belastenden Aussagen Dritter, die unter Folter herbeigeführt worden sind, unterläßt das BVerfG. Es stellt fest:

"Jedenfalls für die hier vorliegende Fallkonstellation, daß ein anderer als der Verfolgte gefoltert wurde, kann eine Fernwirkung des Verwertungsverbots der durch Folter erlangten Aussage auch nicht als elementares rechtsstaatliches Gebot des deutschen Strafverfahrensrechts angesehen werden. (...); soweit in der Literatur eine Fernwirkung befurwortet wird, beschrankt sie dies überwiegend auf die Fälle, in denen es um die Strafverfahren gegen den Beschuldigten geht, der selbst den unerlaubten Vernehmungsmethoden ausgesetzt war. «

Die Chance, die präventive Funktion der Antifolterkonvention auszuweiten und zu stärken, z. B. über eine schlichte Analogie, ist vertan worden. Mutmaßliche Folter an Dritten zur Erlangung von belastenden Aussagen wird nicht ausdrücklich geächtet. Sie entfaltet keine Wirkung auf ein Auslieferungsersuchen. ${ }^{8}$

5 Artikel is der Antıfolterkonvention der Vereinten Nationen sieht als Ausnahme nur die Situation vor, in der eine solche Aussage dazu beitragt, einem mutmaßlıchen Folterer zu beweisen, daß er tatsachlich gefoltert hat.

6 BvR 66/96, S. 14

7 BvR 66/96, S. 17 .

8 Ebenso entfaltete das vor dem Verwaltungsgericht noch anhangıge Asylverfahren des Spanıers keıne Wırkung auf die Auslieferung. 
Seit mehreren Jahren wird die Isolationshaft von Gefangenen, wie sie nach dem bundesdeutschen Recht verhängt werden kann, beanstandet: 9

"Lange andauernde Isolation kann bei den betreffenden Häftlingen schwere physische und psychische Schäden hervorrufen und eine Form grausamer, unmenschlicher und erniedrigender Behandlung darstellen. $\aleph^{10}$

Diese wiederkehrende Formulierung in den ai-Jahresberichten hat nicht dazu geführt, daß über diese Praxis in der breiten Öffentlichkeit diskutiert wurde. ${ }^{11}$

\section{Folter- und Mißhandlungsvorwürfe gegen die Bundesrepublik}

Äußerst empfindlich reagierten Politiker und Verantwortliche auf Vorwürfe über Folterungen durch Polizeibeamte an festgenommenen Personen gegen bundesdeutsche Stellen. ${ }^{12}$ Neben den Fällen von Mißhandlungen waren die Vorwürfe der Folter in dem ai-Bericht konkret auf zwei Fälle bezogen. Auf ausdrückliche Bitte des Vorsitzenden der Gewerkschaft der Polizei, Hermann Lutz, äußerte sich der damalige Vorsitzende der Innenministerkonferenz der Bundesländer, Alwin Ziel aus Brandenburg, gegenüber der Öffentlichkeit wie folgt:

„Zum Thema >Polizei und Ausländer sind wir (die Innenminister der Bundeslander, M.M.-B.) der Auffassung, daß es sich bei Übergriffen der Polizei gegenüber Ausländern, denen ein fremdenfeindliches Verhalten zugeordnet wird, um nicht zu verallgemeinernde Einzelfälle handelt. Undifferenzierte Verallgemeinerungen - wie jungst von amnesty international - schädigen das Ansehen der Polizei und beeinträchtigen das Vertrauen der Bevölkerung in die Polizei insgesamt. $\ll^{13}$

Fünf Monate später veröffentlichte ai einen umfänglicheren Bericht mit dem Titel "Ausländer als Opfer - Polizeiliche Mißhandlungen in der Bundesrepublik Deutschland ${ }^{14}{ }^{14}$ ai bekräftigte die in dem vorherigen Bericht gemachten Vorwürfe und betonte, daß der Organisation in der Zeit von Januar 1992 bis März 199570 Fälle bekannt geworden waren, bei denen deutsche Polizeibeamte in der Ausübung ihres Dienstes in "unverhältnismäßiger und ungerechtfertigter Weise Gewalt angewandt" hätten oder »in Gewahrsam befindliche Personen grausamer, unmenschlicher und erniedrigender Behandlung oder Strafe unterworfen haben sollen«. Der Bericht gelangt in diesem Zusammenhang zu dem Ergebnis, daß der Schwerpunkt der mutmaßlichen polizeilichen Übergriffe gegen Ausländer Berlin ist und die meisten mutmaßlichen Mißhandlungen sowie der behördliche Umgang mit ihnen sich einem »Muster « (pattern) zuordnen lassen. Dieses Muster besteht darin, daß

- die mutmaßlichen Opfer meistens Ausländer oder Angehörige ethnischer Minderheiten sind, die von den Polizeibeamten oft auch mit fremdenfeindlichen Ausdrücken beschimpft werden,

- die in Rede stehenden mutmaßlichen Mißhandlungen schon während oder kurz nach der Festnahme erfolgen,

- die mutmaßlichen Opfer selten sofort umfassend über den Grund für ihre Fest-

9 Die beanstandete Behandlung ist in der Untersuchungshaftvollzugsordnung, also nicht in einem Gesetz, geregelt.

10 amnesty international (Hrsg.): Jahresbericht 1 994, Frankfurt am Main, Fischer Verlag 1994, S. 162.

I I Eine Beschreibung der Wirkung von Isolationshaft gab Birgıt Hogefeld in ihrer Erklarung vor dem Oberlandesgericht Frankfurt am Main am 22.11. 1994 (vgl. taz 25. 1 1. 1994).

12 ai-Index EUR 23/08/94 German.

13 Deutsche Polizei 1/1995, S. 3.

14 ai-Index EUR $23 / 06 / 95$ German. 
nahme und ihre Rechte unterrichtet werden sowie meist keine Möglichkeit erhalten, einen Rechtsbeistand oder Familienangehörige zu unterrichten,

- die erhobenen Beschwerden oder Anzeigen der mutmaßlichen Opfer wegen Mißhandlungen durch Polizeibeamte häufig mit einer Gegenanzeige der beschuldigten Beamten beantwortet werden und schließlich

- viele Verfahren spätestens von der Staatsanwaltschaft unter Hinweis auf sich widersprechende Aussagen der Ausländer und der Polizeibeamten mangels hinreichenden Tatverdachtes gemäß $\$ 170$ Abs. 2 StPO eingestellt wurden und deshalb selten von unabhängigen Gerichten überprüft werden.

Nach der Aufforderung, Mißhandlungsvorwürfe gegen Polizeibeamte künftig häufiger durch unabhängige Gerichte überprüfen zu lassen (nach den $\$ \$$ i $53 \mathrm{ff}$. StPO), schließt der Bericht mit einer Reihe von weiteren Empfehlungen an die Innenminister und -senatoren, die den Mißständen mit Blick auf einzelne Elemente des festgestellten »Musters" wirksam entgegenwirken könnten. In Anschluß an die in Berlin tagende Innenministerkonferenz verteidigte deren Vorsitzender, Alwin Ziel, wie schon im November die bundesdeutschen Polizeibeamten. Er betonte den Einzelfallcharakter der Vorfälle, verwies auf die oft laufenden behördeninternen Ermittlungen oder gerichtlichen Verfahren und befürchtete,

»daß durch die Vorwürfe von amnesty international die Gefahr besteht, daß die Bundesrepublik auf eine Stufe mit Ländern gestellt wird, in denen Folter angewandt wird «. 's

Insgesamt blieben auch alle anderen Erklärungen seiner Kollegen dem Kern der Vorwürfe eigentümlich fern. Ministerielles Bedauern kann offenbar erst nach Erbringung eines schlüssigen Beweises und einem mit einer Verurteilung beendeten Verfahren geäußert werden, obwohl die Vorwürfe im einzelnen selten bestritten wurden.

Auch der Innenausschuß des Deutschen Bundestages verabschiedete am 28.6. I995 unter Zustimmung der SPD einen Entschließungsantrag der Koalitionsfraktionen, in dem er ai indirekt kritisierte und die Figur der »nicht zu verallgemeinernden Einzelfälle« übernahm sowie auf die fehlenden Anhaltspunkte für »rechtsstaatswidriges Verhalten der Untersuchungsstellen « hinwies. Der vorgeschlagene Entschließungstext von Bündnis 9o/Die Grünen, der Schulungsmaßnahmen für die Polizeibeamten forderte, war im Innenausschuß nicht mehrheitsfähig.

$\mathrm{Daß}$ es sich nicht nur um »Einzelfälle" handelt, legten Formulierungen der erst im Januar 1996 bekannt gewordenen und vorher seit längerem unter Verschluß gehaltenen I soseitigen Studie der Polizeiführungsakademie in Münster-Hiltrup nahe, die von der Innenministerkonferenz in Auftrag gegeben worden war. Glaubt man den Presseberichten, so wird in der soziologischen Studie der Führungsakademie die Einzelfall-These ausdrücklich verworfen. ${ }^{16} \mathrm{Am}$ Tag, an dem der neue Vorsitzende der Innenministerkonferenz, der Hamburger Innensenator Wrocklage, ${ }^{17}$ die Studie der Polizeiführungsakademie der Presse vorstellte, untermauerte ai durch einen neuen Bericht die Vorwürfe, bezog Stellung zu den bisher eingegangenen unzureichenden Antworten der politisch Verantwortlichen und erhob neue Mißhandlungsvorwürfe. ${ }^{18}$

Eine bemerkenswerte Wende vollzog daraufhin der neue Innensenator von Berlin, Jörg Schönbohm, der für das Fehlen neuer Mißhandlungsvorwürfe gegen Berliner

15 Pressemitteilung vom i9.5. 1995.

16 dpa 0502 17. I. 1996; ar-Pressemittellung 17. I. 1996; taz 18. I. 1996, 24. I. 1996.

17 Innensenator Wrocklage war erst relativ kurz im Amt. Sein Vorganger, Hackmann, war wegen eines Polizesskandals zuruckgetreten, in dem es um Mißhandlungen von Auslandern durch Beamte der Hamburger Polizei und unzureichende Ermittlungen in diesen Fallen ging.

18 a-Index EUR 23/02/96 German, a1-Pressemittellung 5. 2. 1996 und taz 5. 2. 1996. 
Stellen nicht die begrenzten Ressourcen von ai verantwortlich machte, sondern dies auf die Schulungsmaßnahmen bei der Berliner Polizei zurückführte (Tagesspiegel 7. 2. 1996). Bemerkenswert ist diese Interpretation deshalb, weil sein Vorgänger, Dieter Heckelmann, den ai-Bericht vom Mai 1995 als »einseitig « verworfen und generell die Glaubhaftigkeit von ai in Frage gestellt hatte. Über die Umsetzung der von ai gemachten Empfehlungen zur Verhinderung des Musters von Mißhandlungen durch Polizeibeamte wußte Schönbohm - auch weil er erst kurz im Amt war - nichts zu berichten.

Ähnliche Schwierigkeiten, Menschenrechtsfragen und -politik innenpolitisch zu erörtern, werden auch in dem "3. Bericht der Bundesregierung über dic Menschenrechtspolitik in den auswärtigen Beziehungen « deutlich, der Ende 1995 vorgelegt wurde. Die neu eingeführte ausdrückliche Beschränkung auf die »auswärtigen Beziehungen « ist hierbei der hervorstechendste Makel. Die Kritik vieler Nichtregierungsorganisationen an den beiden vorangegangenen Menschenrechtsberichten der Bundesregierung, die moniert hatte, daß eine glaubhafte und konsistente Menschenrechtspolitik im eigenen Land zu beginnen habe - übrigens ausdrücklich eine Leitlinie der Bundesregierung (vgl. 3. Bericht der Bundesregierung, 1995, S. 7) -, ist durch die neue Bescheidenheit jedenfalls solange nicht ausgeräumt, wie kein innenpolitischer Menschenrechtsbericht in Aussicht gestellt wird. Die Unfähigkeit der Bundesregierung, konsistent und koordiniert zu argumentieren, wird vollends deutlich, wenn am Ende des erwähnten Berichtes »unabhängig vom Bericht« angemerkt wird, daß $»$ über die Asylpraxis und die in diesem Zusammenhang auch unter menschenrechtlich relevanten Gesichtspunkten gemachten praktischen Erfahrungen « der »Bericht des Bundesministers des Innern zur Fortschreibung des Asyl-Erfahrungsberichts 1993 - Asyl-Erfahrungsbericht $1994^{-}$, der dem Innenausschuß des Deutschen Bundestages am 20.6. I994 vorgelegt wurde", Auskunft gebe (3. Bericht der Bundesregierung, 1995, S.60f.). In diesen Berichten des Bundesministers des Innern ist kein Wort zur menschenrechtlichen Dimension der neuen Asylrechtspraxis zu finden.

Als Zwischenbefund bleibt festzuhalten, daß die Bundesrepublik Folter ächtet. Dies bedeutet jedoch nicht, daß die Problematik von Folter oder Situationen, in denen derartige Menschenrechtsverletzungen begangen werden, mit großer Sensibilität bearbeitet werden. ${ }^{19}$ Die Vorwürfe von ai über Folter und Mißhandlungen in der Bundesrepublik werden von den politisch Verantwortlichen allzu oft bagatellisiert oder als ungerechtfertigt und unangemessen verworfen (Polizeibericht des Polizeipräsidiums Köln 3.11.1995, S. 4). Die wenigen Fälle von Mißhandlungsvorwürfen gegen Polizeibeamte, die vor Gericht verhandelt wurden, ergeben kein einheitliches Bild. Immerhin kam es jedoch beispielsweise in dem außergewöhnlich gut dokumentierten Fall Oliver Neß zu zwei Verurteilungen der beschuldigten Beamten. Interessant scheint eine noch nicht vorliegende Revisionsentscheidung des Berliner Kammergerichtes zu sein, die den Freispruch zweier Polizeibeamten durch das Berliner Landgericht mit außerordentlich deutlichen Formulierungen kritisierte (taz (Berlin-Teil) 7. 8. 1996). Darüber hinaus wurden in einem Fall in Köln die Ermittlungen der Staatsanwaltschaft wieder aufgenommen.

Die Haltung zu Folter in der Bundesrepublik ist aber nicht nur dann problematisch,

19 Vgl. amnesty international: 12-Punkte-Programm zur Verhutung der Folter, Oktober 1983. Inweweit die von Alwin Ziel und den anderen Innenministern vorgeschlagene verstarkte Einstellung von Auslandern in den Polizeidıenst sich auf die Vorwurfe auswirken konnte, kann hier nicht beurteilt werden. Anzumerken 1st nur, daß es sich bei dem einen genannten mutmaßlichen Folteropfer um einen bundesdeutschen Burger handelte. al's Vorwurfe der Folterung waren also nicht auf das Feld »Polizei und Auslander « beschrankt, wie Innenmınıster Ziel im o. e. Zitat glauben machen will. 
wenn Vorwürfe gegen die eigenen Institutionen erhoben werden. Es kommt noch eine weitere Dimension des Umgangs mit Folter hinzu, die sich auf die Beurteilung von Folter in anderen Länder bezieht.

\section{Exkurs: Kulturrelativismus versus Unteilbarkeit der Menschenrechte}

Dieser Punkt kann hier nur angedeutet werden. Oft erzeugen Schilderungen über Folter oder Mißhandlungen in anderen Ländern der Welt in der Bundesrepublik auch cine gewisse Ratlosigkeit und Unentschlossenheit. Berichtet man z. B. über die Praxis von unmenschlichen oder grausamen Bestrafungen und deren Opfer, wird zuweilen bei den Zuhörern ein Impuls spürbar, der, anstatt Mitleid, Anteilnahme, Entsetzen oder Hilfsbereitschaft zu artikulieren, eine Art »Ermittlung « einzuleiten beginnt. Im Mittelpunkt des Interesses stehen dann oft nicht mehr die Schrecknisse der Folter, sondern die Frage, ob und inwieweit Folter zu billigen oder zumindest erklärlich ist. Das Entsetzen - so kann man die Reaktion beschreiben - kommt erst danach und damit "zu spät «. Zwischen unmenschliche Tat und dem Entsetzen darüber haben sich rein formalistische, inhaltsleere Legalitätserwägungen geschoben, die dort fest verankert das Entsetzen relativieren. ${ }^{20} \mathrm{Zu}$ beobachten sind Schwierigkeiten, mit den Tatsachen angemessen umzugehen.

In solchen Situationen wird selten der Versuch unternommen, die Mißhandlungen zu leugnen. Vielmehr werden die Voraussehbarkeit des grausamen Strafmaßes oder zumindest die teilweise Verwerflichkeit der bestraften Tat betont und gleichzeitig das »Andere in der (fernen) Kultur bemüht. Heiner Bielefeldt hat darauf aufmerksam gemacht, daß solchen oder ähnlichen Reaktionen oft mindestens zwei Fehleinschätzungen zugrunde liegen: Zum einen werden Menschenrechte für die westliche Welt als seit jeher zur abendländischen Tradition gehörend reklamiert. Dies blendet die bis heute auch in Europa noch stattfindenden Auseinandersetzungen um Inhalt und Verbindlichkeit der Menschenrechte aus. Zum anderen werden die tatsächlichen Probleme, die andere Kulturen mit den Menschenrechten und ihrer Einhaltung haben, bis zu einer strukturellen Unvereinbarkeit hin überzeichnet und dabei den jeweiligen menschenrechtlichen Bemühungen tendenziell kaum Bedeutung beigemessen. ${ }^{21}$

Was hier festgehalten werden soll, ist der oben schon erwähnte Versuch, Distanz zwischen sich und die Folter- oder Mißhandlungsvorwürfe zu bringen. Vor allem wird dabei die Existenz von Folter für Westeuropa nachdrücklich bestritten. ${ }^{22}$ Dabei zu kurz kommen Anstrengungen, dem Kern für einen menschlichen Umgang universelle Geltung zu verschaffen und die eigentlich angezeigte Unterstützung sowie die vorbehaltlose Zusage, Zuflucht für das Folteropfer oder den Gefährdeten zu gewähren.

20 Artikel x (Menschenwurdegebot) in Verbindung mit Artıkel 79 Absatz 3 Grundgesetz (sog. „Ewigkeitsgarantie «) wirkt solchen Prozessen dadurch entgegen, daß ein unabanderlıcher verfassungsrechtlıcher Kern des Grundgesetzes reklamert wird.

21 Bielefeldt, Die Beheimatung der Menschenrechte in unterschiedlıchen Kulturen, in: Bielefeldt, Deıle \& Thomsen (Hrsg.): amnesty international - Menschenrechte vor der Jahrtausendwende. Frankfurt am Main 1996, Fischer Verlag, $173 \mathrm{f}$.

22 Vgl. zur Geschichte: Hobsbawm, Barbareı. Ein Lettfaden. Lettre International, Winter '94, S. $33^{-37}$. 
Aus dem Grundgesetz (Art. I Abs. I und 3) und den oben zitierten internationalen Konventionen ergibt sich ein grundsätzliches Abschiebungsverbot für Personen, denen Folter oder grausame, unmenschliche oder erniedrigende Behandlung in ihrem Heimatland droht. Dem trägt das Ausländergesetz dadurch Rechnung, daß $\$ 53$ Abs. I eine Abschiebung in Fällen drohender Folter verbietet. Das Verbot der Abschiebung bedeutet jedoch nicht, daß der Aufenthalt erlaubt wird. Von Folter Bedrohte werden in der Bundesrepublik lediglich geduldet, ihr Aufenthalt ist nicht rechtmäßig, die Abschiebung gilt lediglich als vorübergehend ausgesetzt. Folteropfer und von Folter bedrohte Flüchtlinge verbleiben fast völlig in der Opportunität des Zufluchtstaates und werden nicht grundsätzlich als Asylberechtigte anerkannt.

\section{I Die »Motivationslehre" des BVerwG}

Ende der siebziger Jahre begann das BVerwG, den verfassungsrechtlichen Begriff der politischen Verfolgung so auszulegen, daß die Begründung des Verfolgerstaates für seine Verfolgungsmaßnahmen maßgeblich für deren "politischen Charakter« und damit die Asylberechtigung wurde. In dem Urteil vom 17.5. 1983 traten dann in Fortführung dieser "Motivationslebre e erstmalig auf höchstrichterlicher Ebene die Begriffe »politische Verfolgung " (damals Art. 16 Abs. 2 S. 2 GG) und »Folter « dogmatisch deutlich auseinander:

»Verletzungen der Menschenwürde - z. B. durch Folter - begründen einen Anspruch auf Asyl nur dann, wenn ihnen politische Motive zugrunde liegen; unberührt bleibt der allgemeine ausländerrechtliche Schutz des Betroffenen. « ${ }^{23}$

Hier wird ein "unpolitischer Folterbegriff « eingeführt, mit der Folge, daß es asylrechtlich möglich wird, gefoltert worden zu sein oder von der Folter bedroht zu sein, ohne in der Bundesrepublik eine Asylberechtigung zu erhalten. Der Flüchtling wird lediglich (vorläufig) nicht abgeschoben, erhält jedoch keine Anerkennung ais politisch Verfolgter und damit keinen dauerhaften Schutz vor Abschiebung in den Folterstaat. Das BVerwG führte in der Begründung zu seiner Motivationslehre aus:

»Für eine Verfolgung aus politischen Gründen kommt es mithin entscheidend auf die den staatlichen Beeinträchtigungen zugrunde liegenden Motive an. (...) Während der Tatbestand der Verfolgung sich aus der Art und Intensitat des Eingriffes ergibt, leitet sich ihr politischer Charakter aus dem Grund für diesen Eingriff (...) oder der ihm zugrunde liegenden Tendenz $(. .$.$) her. Nicht wegen einer in der Schwere der Maßnahme liegenden Verletzung der Men-$ schenwürde, sondern wegen ihrer Motivierung durch personelle Merkmale, (...) wird eine Verfolgung zur politischen. स $^{24}$

Diese, dem juristischen Laien kaum plausibel zu machenden Sätze haben in der Bundesrepublik in den achtziger Jahren zur Versagung von dauerhaftem Schutz für Folteropfer und von Folter Bedrohten geführt. In Fällen von in der Türkei als »extremistisch « eingestuften türkischen Flüchtlingen ging das BVerwG drei Jahre später im Anschluß an seine Motivationslehre noch ein Stück weiter:

"I. Verletzungen der Menschenwürde, wie sie in der Anwendung von Folterpraktiken liegen (hier: während des polizeilichen Ermittlungsverfahrens) begründen einen Anspruch auf Asyl nur dann, wenn sie darauf abzielen, den Betroffenen - zumindest auch - wegen seiner Rasse, Religion, Nationalität, Zugehorigkeit zu einer bestimmten sozialen Gruppe oder wegen seiner politischen Überzeugung zu treffen (...).

23 3. Leitsatz BVerwG g C 36.83 , InfAuslR 1983 , S. 228-235 (228).

24 InfAuslR 1983, S. 228-235 (230), Hervorhebung M.M.-B. 
2. Korperliche Übergriffe, die zur Erzwingung von Aussagen bei Verdacht von politischen wie nichtpolitischen Straftaten unterschiedslos erfolgen, indizieren ihren politischen Charakter nicht. ${ }^{25}$

Neben der 1983 eingeführten »unpolitischen Folter « taucht hier zusätzlich die - so könnte man zugespitzt sagen - strafrechtlich abgesicherte und »ordnungsgemäße ", weil gleichmäßig eingesetzte Folter auf, die kein Asyl in der Bundesrepublik begründen soll. Entscheidend ist zweierlei: Das BVerwG nimmt zum einen strikt die staatliche Perspektive ein. Statt Folteropfer oder von Folter bedrohte Menschen tendenziell in den Begriff der politisch Verfolgten hineinzunehmen, werden sie systematisch von der Asylberechtigung ausgegrenzt. Zum anderen wurde es durch den 2. Leitsatz gerade für Folteropfer schwerer, die asylrechtliche Schwelle zu überschreiten, die Folter von politischer Verfolgung seit dem Urteil trennte. Politisch muß diese restriktive Rechtsprechung vor dem Hintergrund der damaligen »Asyldiskussion « gesehen werden, die abgelehnte Asylbewerber des »Asylmißbrauchs« zieh.

\subsection{Die Einschränkung der "Motivationslebre" durch das BVerf $G$}

Das BVerfG hat in seinem Beschluß vom 20. I2. $1989^{26}$ Einschränkungen der Motivationslehre vorgenommen. Erfreulich deutlich löste sich das Gericht von der staatszentrierten Sichtweise der Bundesverwaltungsrichter ${ }^{27}$ und nahm zumindest teilweise die Perspektive der Bedrohten ein:

»Die subjektiven Grunde und Motive, die den Verfolgenden bei seinen Maßnahmen leiten, sind für die Asylerheblichkeit dieser Maßnahmen nicht von Belang. « $^{28}$

Dieser Perspektivenwechsel hat die Auswirkungen der Motivationslehre des BVerwG auf das Verhältnis von Folter zum Begriff der politischen Verfolgung im Grundgesetz nach immerhin sechs Jahren Geltung stark reduziert. ${ }^{29}$

\subsection{Probleme im Asylverfabren}

Neben den Problemen des materiellen Asylrechts werden die Flüchtlinge, die Folter erlitten oder im Falle einer Abschiebung zu gewärtigen haben, mit verfahrensrechtlichen Hürden konfrontiert. Die Beschleunigungen und die qualitativen Verschlechterungen der Asylverfahren durch Fristverkürzungen und Beschneidungen der Rechtswege in den letzten Jahren sind bekannt. Hier soll nur auf einige Probleme eingegangen werden, die die Auswirkungen deutlich machen. Die rechtlichen Modifizierungen schlagen sich besonders zu Beginn des Asylverfahrens nieder. Neben der einschneidenden Regelung zu sogenannten »sicheren Drittstaaten« (Art. I6a Abs. 2

25 I. und 2. Leitsatz BVerwG 9 C 35.86, InfAuslR 1986, S. $265-269$ (265), Hervorhebung M.M.-B.

262 BvR 958/86, InfAus/R 1990, S. $122-127$.

27 Teilweıse hatten die Bundesverwaltungsrichter in ihren Begrundungen die "Furcht des Staates vor Destabilisıerung der Staats- und Gesellschaftsordnung " von dem staatlıchen $n$ Mißfallen an dem Inhalt der politıschen Überzeugung des Straftaters « zu trennen versucht (zit. n. InfAusIR 1990, S. 125)

28 Zit. n. InfAusiR 1990, S. 125.

29 Zur Vollstandigkeıt soll hier noch angemerkt werden, daß in dem zıtierten Beschluß des BVerfG der Begriff des politısch Verfolgten dahingehend eingeschrankt wurde, daß Asyl nicht beanspruchen konne, wwer im Heimatland unternommene terroristische Aktıvitaten oder deren Unterstutzung von der Bundesrepublik Deutschland aus in den hier moglichen Formen fortzufuhren trachtet." (2. Leitsatz des Beschlusses). Diese Einschrankung kann zwar mıt Blick auf die Meınungsfreiheit von Fluchtlingen in der Bundesrepublik problematısch werden, hat aber in der Asylpraxis bis jetzt keıne bedeutende Rolle gespielt. 
GG), die natürlich auch Folteropfern die Rechtsweggarantie nehmen kann, die aber hier nicht näher beleuchtet werden soll, hat sich vor allem die Zeitspanne zwischen Asylantragsstellung und der ersten nahezu entscheidenden Anhörung verkürzt, in der der Flüchtling seine Verfolgungsgeschichte umfassend, schlüssig und widerspruchsfrei darlegen soll. Der Gesetzgeber wollte gerade in dieser Phase die »tatsächlich Verfolgten" von den Fällen sogenannten "Asylmißbrauchs « trennen. In den ersten drei Monaten sollen die Asylbewerber deshalb grundsätzlich in zentralen Aufnahmeeinrichtungen untergebracht sein. Wie wirken sich diese verfahrensrechtlichen Änderungen in der Praxis auf Folteropfer aus?

Es läßt sich an dieser Stelle nichts Repräsentatives berichten. Deshalb sollen lediglich Eindrücke aus der Flüchtlingsarbeit der letzten Jahre geschildert werden..$^{30}$ Die Flüchtlinge müssen oft völlig unvorbereitet unmittelbar nach ihrer Ankunft in die Anhörung vor dem Bundesamt für die Anerkennung ausländischer Flüchtlinge. Sie wissen regelmäßig nicht, was sie dort genau erwartet und worauf es in einer solchen behördlichen Anhörung tatsächlich ankommt. Aber auch wenn die Verfolgungsgeschichte vorgebracht wird, findet eine angemessene Würdigung durch das Bundesamt oft nicht statt. Im Falle einer Ablehnung durch das Bundesamt wenden sich die Flüchtlinge, oft unter der nicht bekannten Bedingung sehr kurzer Rechtsmittel- und Begründungsfristen im Asylverfahren, an Rechtsanwälte oder Flüchtlingsberatungsstellen.

Die Beobachtungen aus der Praxis gehen dahin, daß diejenigen, die Folter oder Mißhandlungen erlebt haben, oft wenig erzählen - egal, ob sie vor dem Bundesamt oder einer unabhängigen Beratungsstelle stehen. Solche Fälle sind für nicht-professionelle Betreuerinnen und Betreuer deswegen problematisch, da sich dann das Anhörungsprotokoll und der ablehnende Bescheid des Bundesamtes mit ihrem ersten Eindruck decken. Kommt durch Zufall oder aufgrund der Erfahrung der Betreuerinnen oder Betreuer die Folter dann doch zur Sprache, ist der Flüchtling mit den entstehenden Widersprüchen zu seiner ersten Anhörung konfrontiert. Sogenanntes "gesteigertes Vorbringen«, »Unglaubhaftigkeit« oder »Widersprüchlichkeit« sind die Klippen, die wegen rechtlicher Präklusionsvorschriften $(\mathbb{S} 25 \mathrm{Abs} .3$ und 74 Abs. 2 AsylVfG) kaum umschifft werden können, auch wenn die "zweite « Schilderung des Verfolgungsschicksals in sich schlüssig ist. Eine zweite Anhörung zu erreichen oder die Möglichkeit, mit einer im wesentlichen neuen Schilderung das Verwaltungsgericht zu überzeugen, gelingt nur selten. So kommt es immer wieder zu Fällen, in denen Menschen nach ihrer Abschiebung aus der Bundesrepublik nachweislich gefoltert werden. ${ }^{31}$ Oder Folteropfer ${ }^{32}$ sollen wieder in den Folterstaat abgeschoben werden, weil ihr Asylantrag als »offensichtlich unbegründet« abgelehnt worden war. Zusammenfassend läßt sich sagen, daß der Gesetzgeber in seinem Bemühen, die »tatsächlich Verfolgten « von den sogenannten Asylmißbrauchsfällen zu trennen, durch die Ausgestaltung des Asylverfahrens gerade die äußerst verletzbare Gruppe der Folteropfer trifft.

30 Vgl. auch Republıkanıscher Anwaltsvereın (Hrsg.): Umgang mıt Folteropfern im Asylverfahren, Berlin 1996.

31 Urgent Actions (UA 408/94-1; ai-Index: EUR 44/136/94 und EUR 44/1 50/94) zum Fall Murat Fani, einem Kurden aus der Turkei, 18.11. 1994, und 8. 12. [994, oder grundsatzlıcher die ai-Stellungnahme zur Gefahrdung von Kurden im Falle ihrer Abschebung in die Turkei, 19. 7. 1996.

32 Urgent Action (UA Extra r1/95; a1-Index: EUR 23/01/95) zum Fall Fariz Simsek, einem Kurden aus der Turkei, 30. 1. 1995 . 
Die von der Bundesrepublik ausgesprochene außenpolitische Ächtung der Folter ist grundsätzlich zu begrüßen. Es fehlt in diesem Bereich weniger an der Akzeptanz der völkerrechtlichen Instrumente oder der damit einhergehenden Menschenrechtsrhetorik als vielmehr an Konsistenz, Konfliktfähigkeit und einem nachvollziehbaren Verhältnis von menschenrechtlicher Verantwortung und machtpolitischen Interessen - vor allem in Situationen der Konfrontation mit Staaten, die Folter anwenden. Reine außen- und wirtschaftspolitische Opportunitätserwägungen der Bundesregierung lassen sich oft kaum von ihren Bemühungen in »stiller Menschenrechtsdiplomatie* trennen.

Noch problematischer stellt sich die zu schwache innen- und asylpolitische Reflexion der Phänomene grausamer oder unmenschlicher Behandlung und Folter dar. Aus der außenpolitischen Ächtung folgt bis heute weder eine angemessene Diskussion über die Vorwürfe von Folter oder Mißhandlungen durch Polizeibeamte noch ein dauerhafter und effektiver Schutz für Flüchtlinge vor Abschiebung in einen Staat, der Folter anwendet. Zum einen werden Vorwürfe gegen bundesdeutsche Stellen von den politisch Verantwortlichen angezweifelt, kritisiert und unzureichend bearbeitet. Zum anderen treffen die Asylrechtsänderungen der letzten Jahre Folteropfer besonders hart und belassen sie darüber hinaus oft in einem Zustand der Schutzlosigkeit.

\section{Manfred H. Wiegandt Ein Satyrspiel um die Abgeordnetenimmunität*:}

Für Anfang August 1996 waren die sog. "Chaos-Tage« angekündigt, mit denen Punks wie bereits im Jahr 1995 in der niedersächsischen Landeshauptstadt zu einem mehrägigen Happening zusammenkommen wollten. Nach den Erfahrungen des Vorjahres, bei denen Bilder von Barrikaden und Bränden, von steinewerfenden und öffentlich urinierenden Punks in der Innenstadt Hannovers in den Medien die Runde gemacht hatten, waren sich Stadt und Landkreis Hannover, Polizeiführung und niedersächsische Landesregierung im letzten Sommer einig, daß sich solche Vorkommnisse nicht wiederholen dürften. Die "Chaos-Tage « wurden im vorhinein durch Allgemeinverfügung nach $\$$ is Vers $G$ verboten. Gegen Personen, die sich nach ihrem äußeren Erscheinungsbild, sprich auffällige Kleidung oder Haartracht, als mögliche Teilnehmer an den verbotenen »Chaos-Tagen « auswiesen, sollte die Polizei mittels eines präventiven Aufenthaltsverbotes/Platzverweises gem. dem erst kürzlich reformierten $\$$ I7 II I NGefAG vorgehen. ${ }^{1}$ Im Vorfeld des polizeilichen Einsatzes nannte die niedersächsische Landtagsabgeordnete des BÜNDNIS 9o/Die GRÜNEN, Silke Stokar von Neuforn, die in diesem Zusammenhang ergangene Verbotsverfügung laut einem Pressebericht eine "Realsatire " und bezeichnete sie als eine

* Meinem Anfang des Jahres todlich verungluckten Studienfreund, Rechtsanwalt Ullrich Affelt (Hannover), in memoriam.

I Siehe dazu Jurgen Seifert, Chaos-Tage: Modell Hannover. Aufenthaltsverbote zur Durchsetzung von Versammlungsverboten?, KJ $1996,356-36$ r. 